\title{
Effect of spatial spectrum overlap on Fourier ptychographic microscopy
}

\author{
Qiulan Liu*, Cuifang Kuang*,†, ${ }^{*}$, Yue Fang*, Peng Xiu*, Yicheng Li*, \\ Ruixin Wen* and $\mathrm{Xu} \mathrm{Liu}{ }^{*, \dagger}, \S \uparrow$ \\ *State Key Laboratory of Modern Optical Instrumentation \\ College of Optical Science and Engineering \\ Zhejiang University \\ Hangzhou 310027, P. R. China \\ ${ }^{\dagger}$ Collaborative Innovation Center of Extreme Optics \\ Shanxi University, Taiyuan, 030006, P. R. China \\ *cfkuang@zju.edu.cn \\ §iiuxu@zju.edu.cn
}

Received 28 February 2016

Accepted 18 April 2016

Published 8 June 2016

\begin{abstract}
Fourier ptychographic microscopy (FPM) is a newly developed imaging technique which stands out by virtue of its high-resolution and wide FOV. It improves a microscope's imaging performance beyond the diffraction limit of the employed optical components by illuminating the sample with oblique waves of different incident angles, similar to the concept of synthetic aperture. We propose to use an objective lens with high-NA to generate oblique illuminating waves in FPM. We demonstrate utilizing an objective lens with higher NA to illuminate the sample leads to better resolution by simulations, in which a resolution of $0.28 \mu \mathrm{m}$ is achieved by using a high-NA illuminating objective lens $(\mathrm{NA}=1.49)$ and a low-NA collecting objective lens $(\mathrm{NA}=0.2)$ in coherent imaging $(\lambda=488 \mathrm{~nm})$. We then deeply study FPM's exact relevance of convergence speed to spatial spectrum overlap in frequency domain. The simulation results show that an overlap of about $60 \%$ is the optimal choice to acquire a high-quality recovery $(520 * 520$ pixels) with about 2 min's computing time. In addition, we testify the robustness of the algorithm of FPM to additive noises and its suitability for phase objects, which further proves FPM's potential application in biomedical imaging.
\end{abstract}

Keywords: Fourier ptychographic microscopy; synthetic aperture; convergence speed; spatial spectrum overlap.

\Corresponding authors.

This is an Open Access article published by World Scientific Publishing Company. It is distributed under the terms of the Creative Commons Attribution 4.0 (CC-BY) License. Further distribution of this work is permitted, provided the original work is properly cited. 


\section{Introduction}

To break the diffraction limit and capture more details of the sample, lots of efforts have been made. Many tremendous accomplishments in this area have been achieved since 1990s. In recent years, researchers have proposed a great many super-resolution methods, such as stimulated emission depletion microscopy (STED), ${ }^{1}$ photoactivated localized microscopy (PALM $),{ }^{2}$ stochastic optical reconstruction microscopy (STORM), ${ }^{3}$ and so on.

Apart from these nanoscopy techniques, another researching field is to acquire the high spatial information of the object via distinct illumination. Among these methods, great attention has been attached in structured illumination microscopy (SIM). ${ }^{4}$ In addition to SIM, the concept of illuminating the sample at oblique angles and synthetically increasing the numerical aperture of the objective lens has been developed, which is termed synthetic aperture imaging. ${ }^{5-8}$ It was derived from radar technology $y^{9}$ and found wide application in optical phase imaging. ${ }^{10-14}$

Fourier ptychographic microscopy $(\mathrm{FPM})^{15-21}$ is a newly developed imaging technique using the concept similar to synthetic aperture to improve the system's imaging performance beyond the limit introduced by its optical components. The algorithm of FPM originates in the Gerchberg-Saxton (GS) algorithm, ${ }^{22}$ which is one of the earliest strategies for recovering a sample's image. The basic idea of FPM is expanding the spatial spectrum (i.e., Fourier spectrum) in frequency domain via multiimage fusion. FPM converges to a high-resolution image by constraining the amplitude of the solution using the acquired low-resolution images. The accuracy and reconstructing speed of FPM recovery depend on the amount of spatial spectrum overlap. In this paper, the primary goal is to explore the exact relationship between convergence speed and data redundancy. In addition, we further discuss the FPM's imaging performance with regard to different noises and the ability of recovering the intensity of a sample with random phase.

\section{Principle of Resolution Enhancement with Synthetic Aperture}

Because of the diffraction effect induced by the finite size aperture of the objective lens, the ability of the microscopy to acquire fine details or the highest spatial frequency of the object is limited. According to the Abbe criterion, ${ }^{23}$ the resolution of the conventional microscopy in coherent imaging is roughly

$$
R=\frac{\lambda}{\mathrm{NA}},
$$

where $\lambda$ is the wavelength of the incident light and $\mathrm{NA}$ is the numerical aperture of the objective lens.

The objective lens of microscope can be regarded as a low-pass filter. In the frequency domain, the spatial frequencies are filtered by this low-pass filter whose pupil function (i.e., coherent transfer function) can be described as

$$
P(\xi, \eta)=\operatorname{circ}\left(\frac{\sqrt{\left(\xi /(\lambda f)^{2}\right)+\left(\eta /(\lambda f)^{2}\right)}}{\mathrm{NA} / \lambda}\right),
$$

where $f$ is the focal length of the objective lens.

As shown in Fig. 1(a), consider a plane wave with the wave vector $\left(k_{x}^{i}, k_{y}^{i}\right)$ (or $\left.2 \pi\left(v_{x}^{i}, v_{y}^{i}\right)\right)$ like Eq. (3) (the subscript $i$ denotes $i$ th illuminating light beam)

$$
\begin{aligned}
U_{\text {in }}^{i}\left(x_{0}, y_{0}\right) & =\exp \left[-j\left(k_{x}^{i} x_{0}+k_{y}^{i} y_{0}\right)\right] \\
& =\exp \left[-j 2 \pi\left(v_{x}^{i} x_{0}+v_{y}^{i} y_{0}\right)\right],
\end{aligned}
$$

to illuminate the sample under an oblique angle $\theta_{i}$, the complex amplitude at the pupil plane (i.e., Fourier plane) becomes ${ }^{24}$

$$
\begin{aligned}
U_{f}^{i}(\xi, \eta)= & P(\xi, \eta) \frac{1}{j \lambda f} \iint o\left(x_{0}, y_{0}\right) U_{\mathrm{in}}^{i}\left(x_{0}, y_{0}\right) \\
& \times \exp \left[-j 2 \pi\left(\frac{\xi}{\lambda f} x_{0}+\frac{\eta}{\lambda f} y_{0}\right)\right] d x_{0} d y_{0}, \\
= & \frac{1}{j \lambda f} P(\xi, \eta) O\left(\frac{\xi}{\lambda f}+v_{x}^{i}, \frac{\eta}{\lambda f}+v_{y}^{i}\right)
\end{aligned}
$$

where $O(\xi /(\lambda f), \eta /(\lambda f))$ is the Fourier transform of the object function $o\left(x_{0}, y_{0}\right)$. From Eq. (4), we can see that the obliquely incident plane wave with wave vector $\left(k_{x}^{i}, k_{y}^{i}\right)$ forces the center of the object's spatial spectrum a frequency shift by $\left(k_{x}^{i}, k_{y}^{i}\right)$, which results in the low-pass filter covering a different region of the spectrum. Therefore, tilted illumination provides an approach for shifting higher frequencies of the object into the collection cone of the collecting objective lens (OL2). In other words, scattered light from the object beyond the NA of OL2 which contains the fine details of the object can be detected, thus offering the opportunity for improving the resolution of the system.

Figure 1(a) shows a suitably designed scanning strategy for such a synthetic aperture. What is 


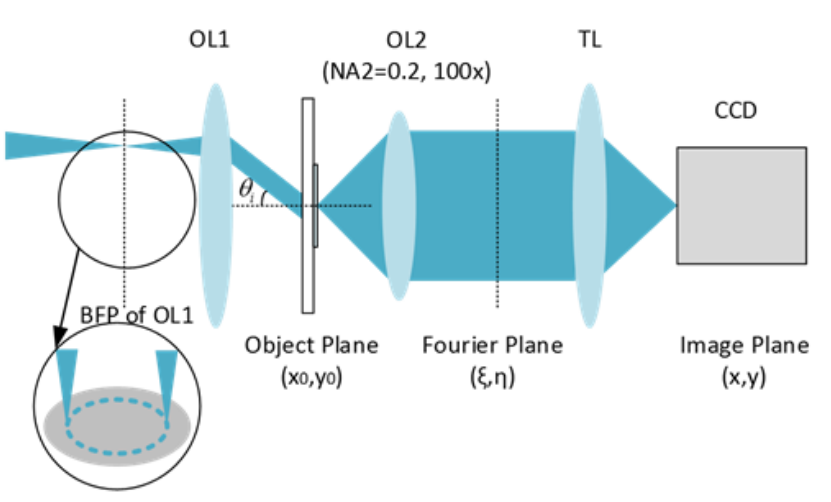

(a)

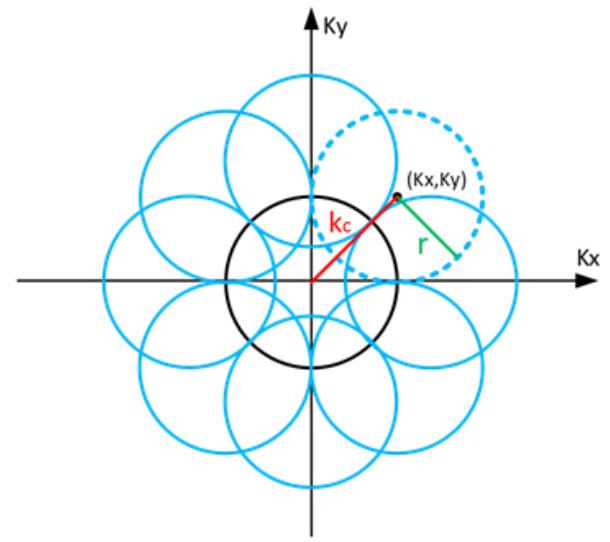

(b)

Fig. 1. Schematic of synthetic aperture. (a) Schematic diagram of synthetic aperture by tiled illumination to improve the resolution of the microscope. (b) Synthesis of spatial spectrum in the pupil plane.

novel is that we employ a high-NA objective lens (OL1) to generate the illuminating light. An illuminating light beam scans the back focal plane (BFP) of the objective lens for illumination and the focused point on the BFP can be scanned circularly on BFP, as is depicted in the inset of Fig. 1(a), thus producing obliquely incident plane waves to illuminate the object under various angles. As is presented in Fig. 1, the scanning radius corresponds to the incident angle $\theta_{i}$, which causes a frequency shift of the object's spatial spectrum by $k_{c}=k \sin$ $\left(\theta_{i}\right)=(2 \pi / \lambda) n_{1} \sin \left(\theta_{i}\right)$ in Fourier plane, where $\lambda$ and $n_{1}$ are wavelength in the free space and the refractive index between OL1 and object, respectively. The size of a single circular object's spatial spectrum passing through the system depends on the NA of OL2, whose radius corresponding to the cutoff spatial frequency can be described as $r=(2 \pi / \lambda)$ NA2, where NA2 is the numerical aperture of OL2 determining the very resolution of the conventional microscope. When the illuminating light beam scans at the BFP of OL1 one circle by one circle, different regions of the object's spatial spectrum shown in Fig. 1(b) will be acquired. If we sum over all the small circular object's spatial spectrum, an expanded spectrum containing highfrequency components of the object will be obtained.

However, it's worth noting that taking a direct sum of the spectrum is not accurate enough, because a direct sum would place too much emphasis on the low frequencies and introduce aberrations. ${ }^{25}$ Here, we employ the computational method of FPM. Its main principle is iteratively stitching together a set of low-resolution images in frequency domain to recover an accurate high-resolution image; more details about FPM are given in Sec. 3 .

The final resolution of the microscope is determined by the size of the expanded spatial spectrum we will capture in frequency domain, i.e., it relies on how large we can acquire the value of $k_{c}$, which corresponds to the largest incident angle $\theta_{\max }$ of the illumination when the calculation is mapped to the spatial domain. Therefore, OL1 as the illuminating objective lens plays a significant role in improving the resolution of the system. It's not difficult to find that NA1 (number aperture of OL1) dominates the final resolution of the microscope if we fix the NA of OL2. The maximum shifting of frequency can be written as $k_{c \max }=(2 \pi / \lambda) n_{1} \sin \left(\theta_{\max }\right)=(2 \pi / \lambda) \mathrm{NA} 1$.

A simulation has been made by adopting the FPM calculating method with the synthetic aperture concept mentioned above. As is depicted in Fig. 2, different NAs of OL1 are employed to demonstrate the relationship between resolution improvement and NA of OL1. Figure. 2(a) shows the FP recovery result of a spoke-like sample with the NAs changing from 0.7 to 1.49. The refractive index of the immersion OL1 is 1.52. The corresponding expanded spatial spectra of Fig. 2(a) captured in frequency domain are shown in Fig. 2(b). We can see that with the growth of NA1, the captured spatial spectra enlarges, and at same time the corresponding resolution of the system also improves gradually. The cutoff spatial frequency in the spatial frequency space becomes $(2 \pi / \lambda)$ NA1 $+(2 \pi / \lambda) \mathrm{NA} 2=(2 \pi / \lambda)(\mathrm{NA} 1+\mathrm{NA} 2)$ by introducing a frequency shift of $(2 \pi / \lambda)$ NA1. Therefore, the ultimate resolution of the optical microscope acquired 


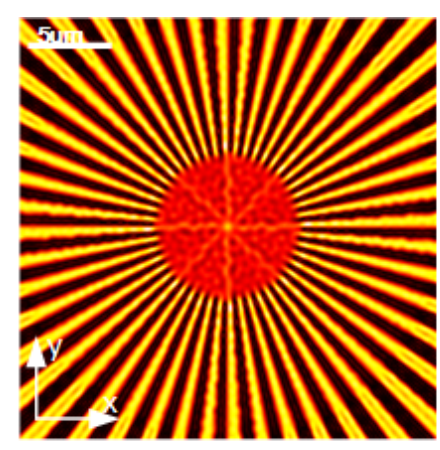

(a1)

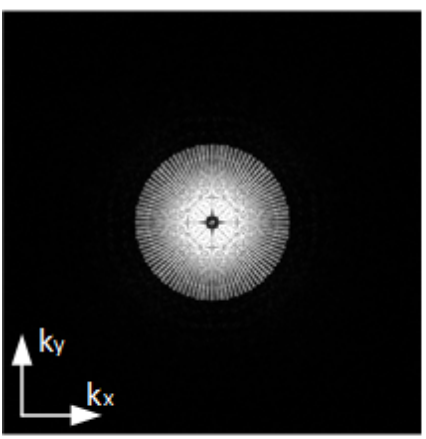

(b1)

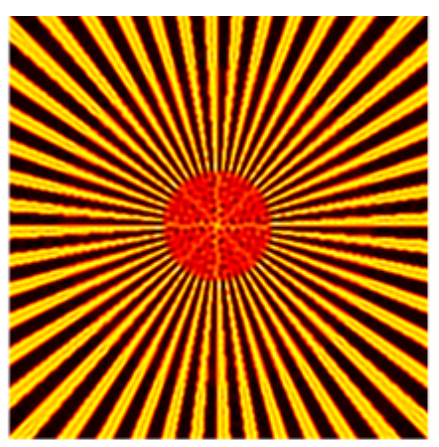

(a2)

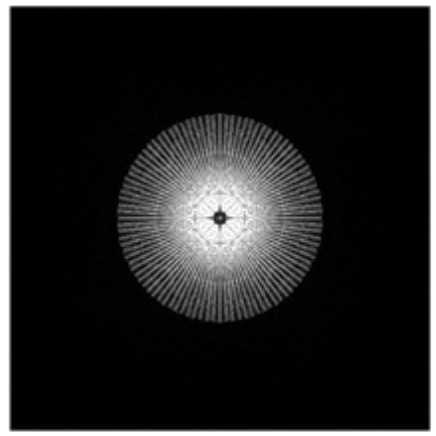

(b2)

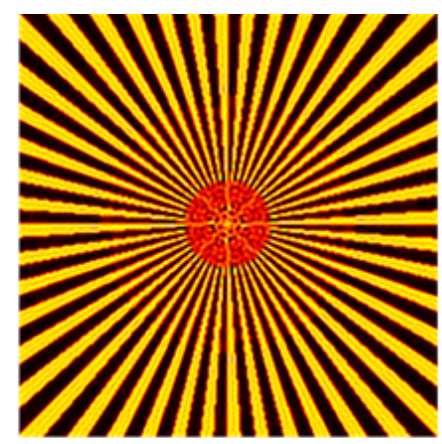

(a3)

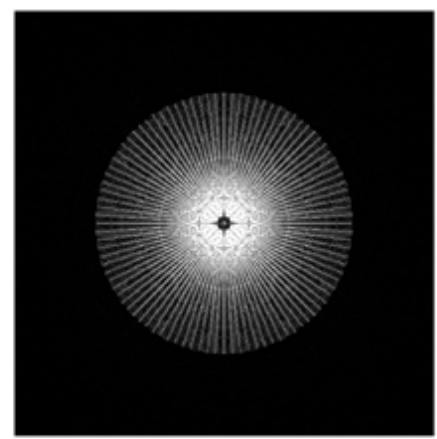

(b3)

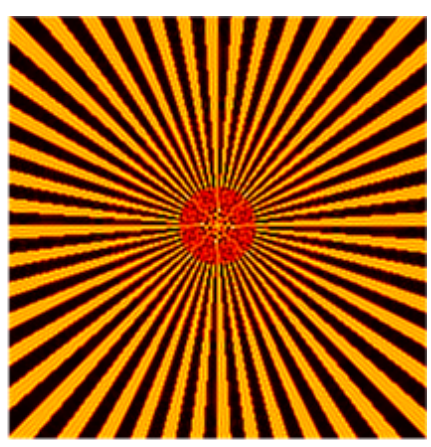

(a4)

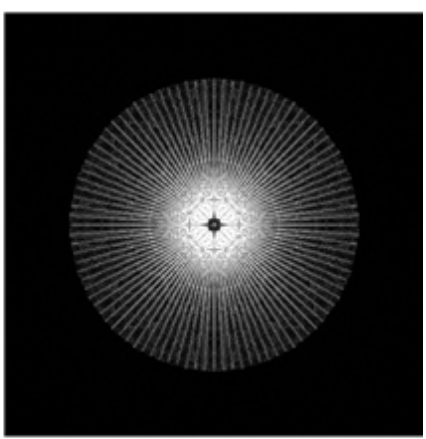

(b4)

Fig. 2. FPM recovery using different NA of OL1. (a) Simulated results of a spoke-like sample with different NA of OL1, the NAs are (a1) 0.7, (a2) 1, (a3) 1.3, and (a4) 1.49, respectively. (b) The corresponding spatial spectra of (a). The scale bar is $5 \mu$ m.

can be described as

$$
R=\frac{\lambda}{N A 1+N A 2} .
$$

Therefore, to obtain higher resolution of the system, a higher NA1 and NA2 can be chosen to achieve this end. Nevertheless, since the working distance and FOV of high NA objective lens is usually limited, in this case, we use a low-NA OL2 allowing a large FOV to be obtained at the expense of a low spatial resolution. Meanwhile, a high-NA OL1 is employed to improve the final resolution by means of FPM. In this paper, NA1 is equal to 1.49 and NA2 is chosen to be 0.2 . Thus, by applying the reconstruction algorithm of FPM to the synthetic aperture technique, we transform a conventional optical microscope into a high-resolution $(0.28 \mu \mathrm{m})$ microscope.

\section{Algorithm of FPM}

A review of FPM's reconstruction algorithm will ${ }^{15}$ be helpful to understand the principles of resolution enhancement. FPM is a coherent imaging technique that uses various angle illumination for recovering a high-resolution image, which switches between spatial $(x-y)$ and frequency $\left(k_{x}-k_{y}\right)$ domains. In the spatial domain, the obtained images are used as a supporting constraint for the amplitude of the solution. In the frequency domain, the pupil function of OL2 is applied to constrain the solution.

A flow chart of the FPM's algorithm is shown in Fig. 3. It begins with an initial guess of the object profile and the corresponding spatial spectrum (step 1 ). This initial spectrum is sequentially updated by the corresponding region of Fourier spectrum of the replaced intensity image (step 2). The iterative process is repeated until the solution converges (step 3).

The three steps elaborated in a more detailed way are as follows: Step 1: it starts with an initial guess of the high-resolution object profile $I_{h}$ and the obtained corresponding spatial spectrum. Here, we choose the image captured under normal illumination as the initial intensity guess. Step 2.1: select a subregion of the initial spectrum whose area is equal to low-pass band of the low-NA collecting objective lens. The central position $\left(k_{x}^{1}, k_{y}^{1}\right)$ of the spectral subregion corresponds to the illuminating angle. Then inverse Fourier transform of the selected spectrum is performed to generate a low-resolution image $\sqrt{I_{1}} e^{i \varphi_{1}}$. 

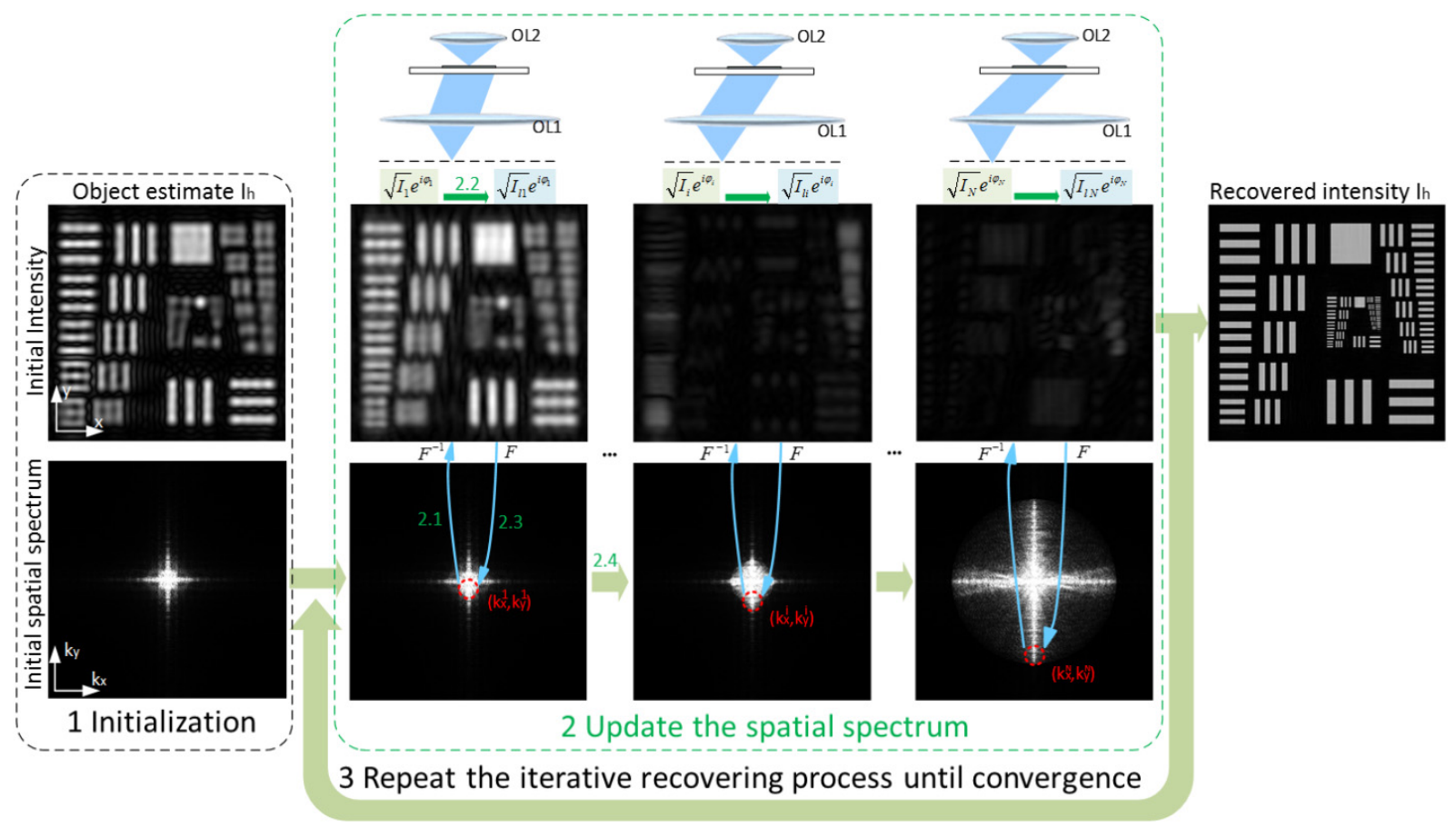

Fig. 3. Flow chart of FPM's algorithm. A high-resolution intensity image is recovered by using $N$ low-resolution intensity images $I_{l i}$ which are obtained under variable incidence of illumination. Steps 1-3 illustrate the iterative recovering process of FPM. Step 1: initialize the object estimate $I_{h}$. Step 2.1: select a subregion of the initial spatial spectrum and perform inverse Fourier transform to generate a low-resolution image $\sqrt{I_{1}} e^{i \varphi_{1}}$. Step 2.2: replace $I_{1}$ by the recorded image intensity $I_{l 1}$. Step 2.3: update the corresponding subregion of $\sqrt{I_{l 1}} e^{i \varphi_{1}}$ in frequency domain (the region encircled by the red circle). Step 2.4: repeat steps 2.1-2.3 for other plane-wave incidences (total of $\mathrm{N}$ intensity images). Step 3: repeat steps 2 until converges.

Step 2.2: the intensity $I_{1}$ of the target image is replaced with the intensity of low-resolution record $I_{l 1}$ captured under the corresponding illuminating angle, forming an updated target image $\sqrt{I_{l 1}} e^{i \varphi_{1}}$. Step 2.3: The selected subregion of the spatial spectrum is replaced with the corresponding Fourier transform spectrum of the updated image $\sqrt{I_{l 1}} e^{i \varphi_{1}}$. Step 2.4: steps 2.1-2.3 are applied to the recorded image under $i$ th illuminating angle until the total $N$ recorded images are all performed the above steps 2.1-2.3. Step 3 : the iterative process of step 2 is repeated until the solution converges, and finally we acquire the recovered high-resolution image $I_{h}$. The convergence is estimated by the mean-square-error (MSE) of two consecutively recovered solutions. The process sustains until the MSE is smaller than a pre-set value.

\section{Simulation Results and Analysis}

\subsection{The relationship between conver- gence speed and spectrum overlap}

The validity of FPM has been clarified in nature. ${ }^{15}$ In this section simulations have been made to demonstrate a relationship between a spatial spectrum overlap and convergence speed in this iterative process of FPM. In step 1 and 2, the space between the centers of the selected adjacent circular spectra is not fixed, in other words, the overlapping area between the two adjacent spectra is flexible. Figure 4 shows two different overlapping cases with the same expanded spectrum in frequency domain. In Fig. 4(a), we update the spectrum with a large overlapping area, while in Fig. 4(b), the overlapping

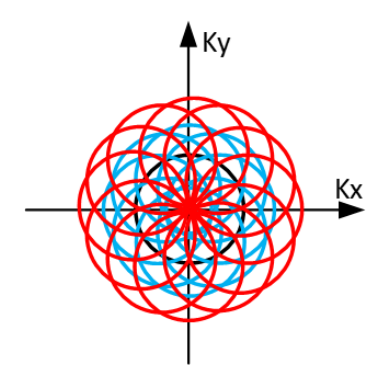

(a)

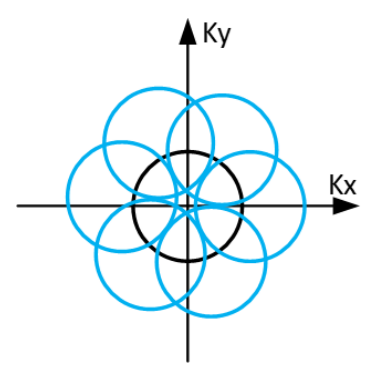

(b)
Fig. 4. Two different overlapping cases of spectrum in frequency domain. The overlapping area between the two adjacent circular spectra is about (a) $75 \%$, and (b) $25 \%$. 
Q. Liu et al.

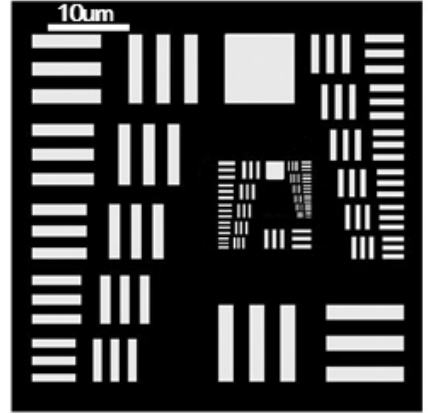

(a)

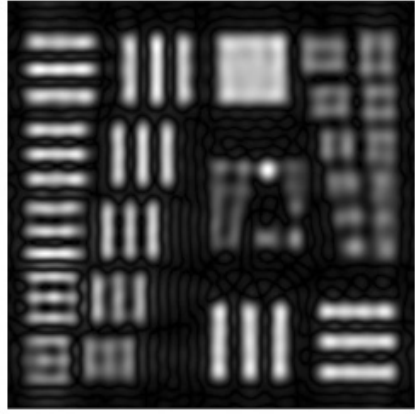

(b)

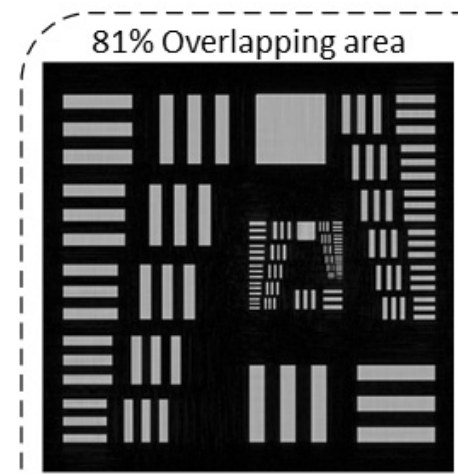

(c1)

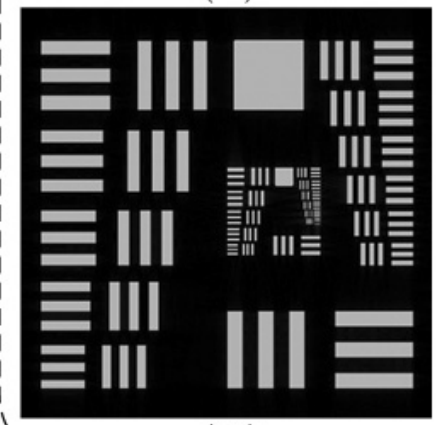

(d1)

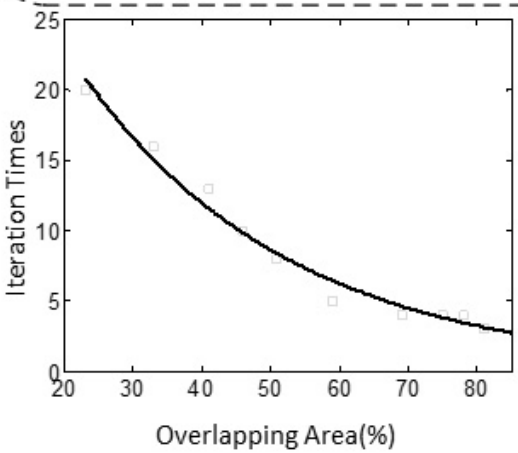

(e)

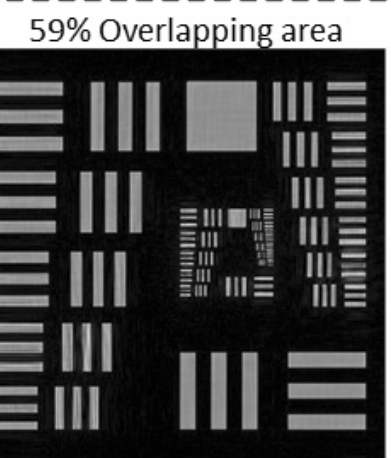

(c2)

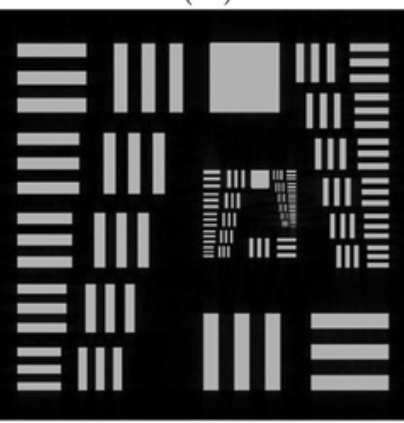

(d2)

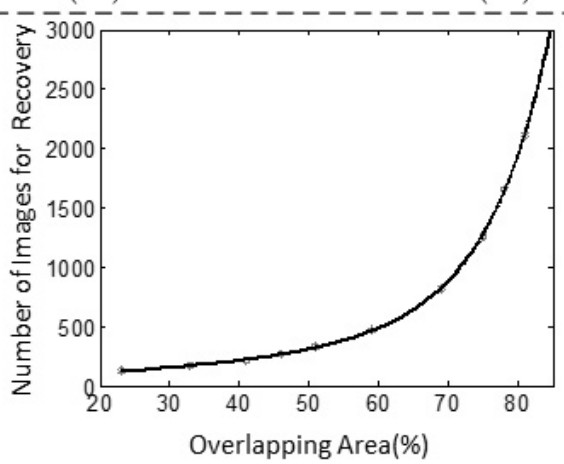

(f)

(c3)

(d3)
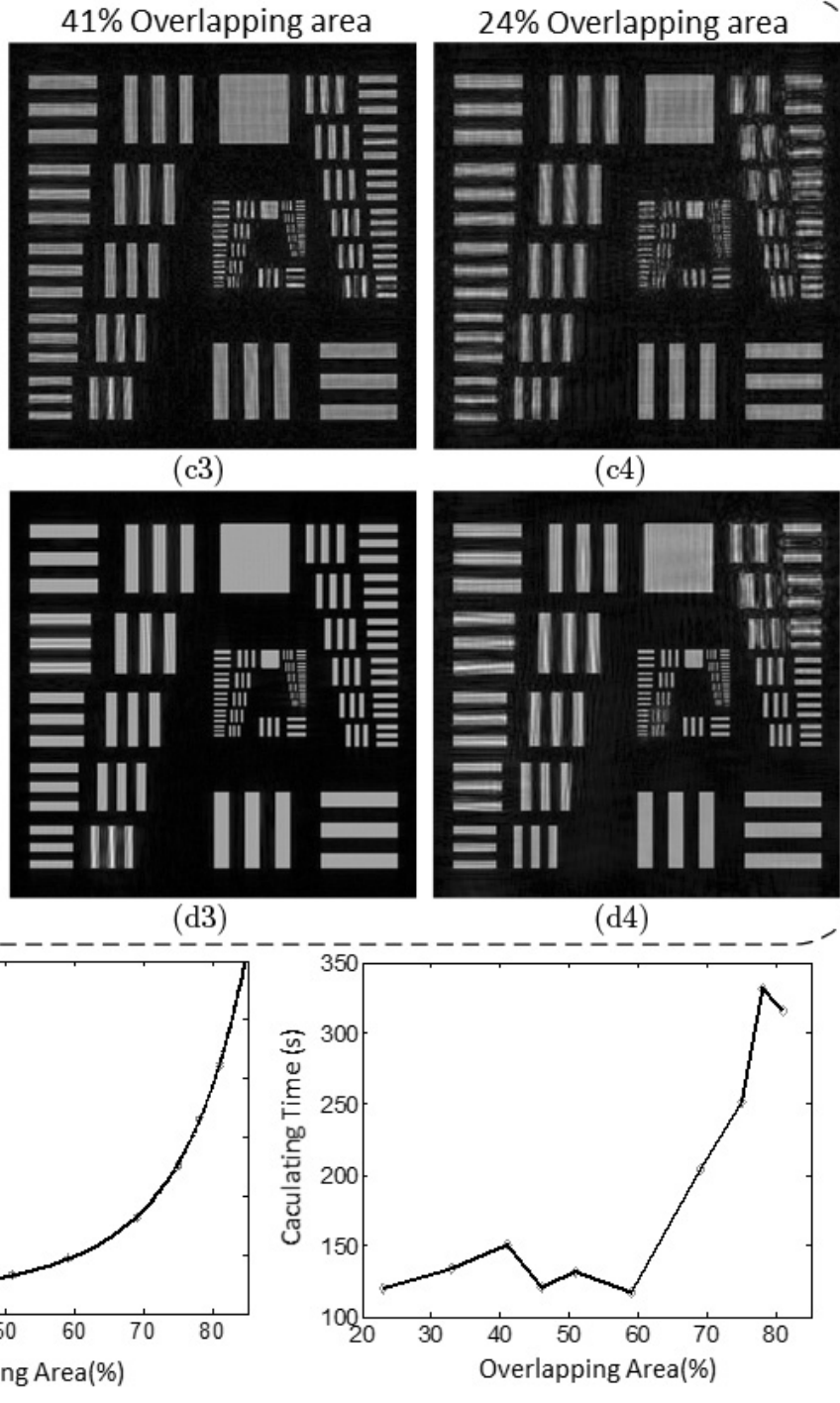

(g)
24\% Overlapping area

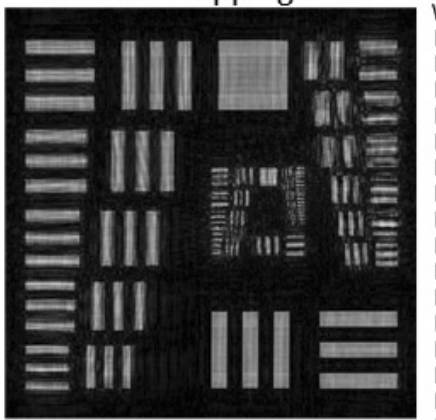

(c4)

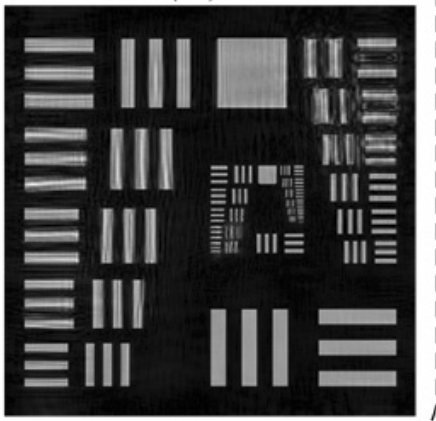

(d4)

Fig. 5. Recovery of FPM with different overlapping area of spectrum. (a) The input raw image of resolution target. (b) Obtained image with normal illumination. FPM recovered intensity by iterating (c) for once, and (d) until convergence, with 1.49 illuminating NA and 0.2 collecting NA applied. The corresponding overlapping areas for (c1)-(c4) and (d1)-(d4) are $81 \%, 59 \%, 41 \%$ and $24 \%$, respectively. (e) The relationship between overlapping area and iterative times. (f) The number of images for FPM's recovery is plotted as a function of overlapping area. $(\mathrm{g})$ The relationship between calculating time and overlapping area. 
area is very small. With these different overlapping cases, the iterative times or the convergence speed of FPM's recovering progress displays some regularity.

Figure 5 shows the FPM recovered intensity of the resolution target with different overlapping cases in the frequency domain. As is depicted in Fig. 5(b), when the raw sample (see Fig. 5(a)) is illuminated under a normal incidence, the fine details of the acquired image can hardly be distinguished. The resolution improves significantly if we introduce the strategy of FPM, which is just as shown in Figs. 5(c) and 5(d). Four overlapping cases were chosen to demonstrate the relationship between iterative times and overlapping area. The overlapping areas are $81 \%, 59 \%, 41 \%$ and $24 \%$ respectively corresponding to Figs. 5(c1)-5(c4) and $5(\mathrm{~d} 1)-5(\mathrm{~d} 4)$. Figure 5(c) shows the FPM's recovery iterated for only once. We can see that with the overlapping area decreasing, the performance of FPM reconstruction becomes worse. In Fig. 5(d), the reconstructing procedure sustains until the solution converges. As is depicted in Fig. 5(d2)-5(d4), the more the adjacent circular spectra overlaps, the higher similarity to the sample the FPM's recovery will be acquired. The iterative times for converging shown in Figs. 5(d1)-5(d4) are 3 with $81 \%, 5$ with $59 \%, 14$ with $41 \%$, and 19 with $24 \%$ overlap, respectively. Figure 5(e) shows the corresponding relationship between iterative times and overlapping area, in which we can find that the iterative times decreases exponentially with the growth of overlapping area. However, large overlapping area means a great number of low-resolution images acquired by various angles for illuminating are needed to reconstruct a high-resolution image of the sample. Figure 5(f) demonstrates the relationship between the number of images needed for FPM recovery and the overlapping area. The speed of acquiring those low-resolution images is determined by the scanning speed of the illuminating light beam. As for the calculating speed, the processing time for 100 low-resolution images is about $5 \mathrm{~s}$ in Matlab using a personal computer with an Intel i7 CPU. The relationship between the calculating time of acquiring a high-resolution image and the overlapping area is shown in Fig. 5(g). In consideration of the high-quality of recovery and the computing

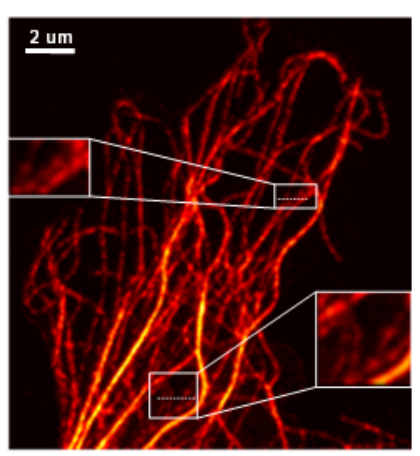

(a1)

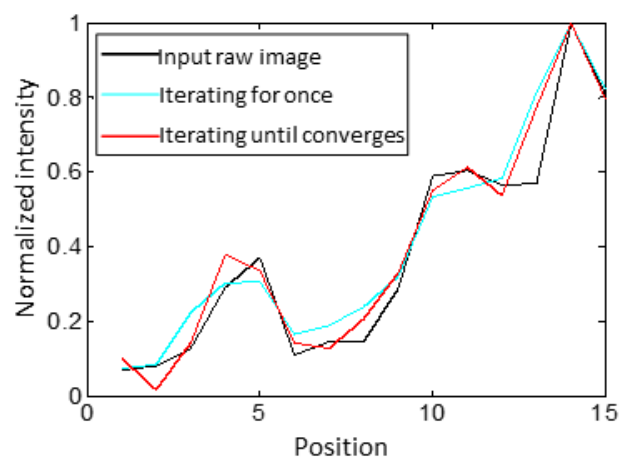

(b1)

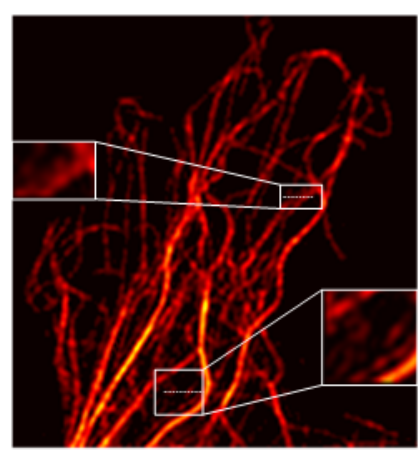

(a2)

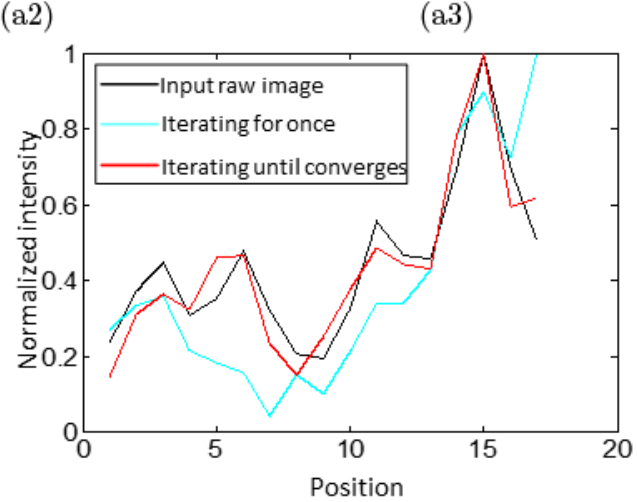

(b2)

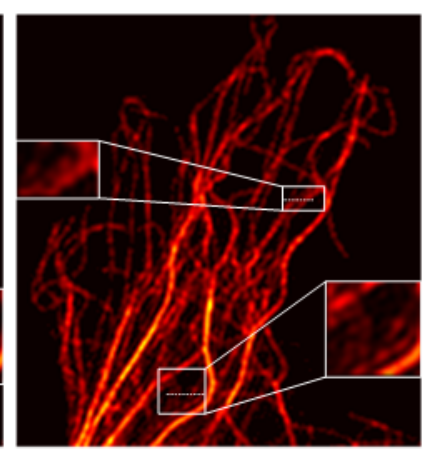

(a3)

Fig. 6. Simulation results for a microtubule with 59\% overlapping area in frequency domain. (a1) The input raw image of the sample. (a1)-(a2) FPM's recovery iterated for only once and five times until the solution converges, respectively. (b1)-(b2) Intensity profiles alone the upper and lower white dotted lines in (a), respectively. 
speed, the overlapping area is good enough chosen to be about $60 \%$, in which the high-resolution image is obtained by about $2 \mathrm{~min}$.

To clearly demonstrate the resolution enhancement and the relationship between converging speed and overlapping area using the combination of synthetic aperture technique and algorithm of FPM, we also performed a simulation using a biological sample. Figure 6 presents the simulation results where the overlapping area is $59 \%$, from which we proved once again the resolution improvement of the proposed method. As the red lines shown in Figs. 6(b1)-6(b2), with the increasing of iterative times, the contrast of the image will improve correspondingly, and here five times of iteration is quite enough to reconstruct a high-resolution image with regard to a spatial spectrum overlap of $59 \%$.

\subsection{Imaging performance in the presence of additive noises}

We also investigated the imaging performance robustness to noises of FPM. Figure 7 demonstrates the performance to the Gaussian noise by simulating a spoke-like sample. In Fig. 7(a), the images without noise are acquired until the solution converges, where the iterative times are 3, 5, 14 and 19 for Fig. 7(a1)-7(a4), respectively. In Figs. 7(b)-7(c),

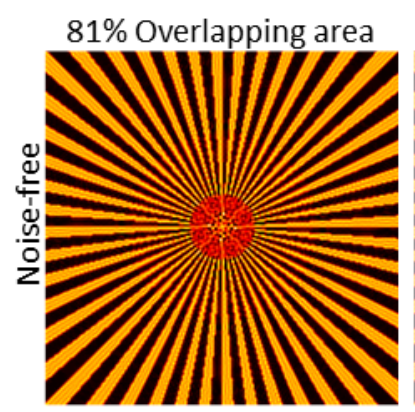

(a1)

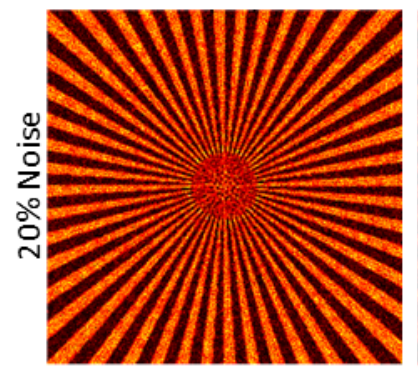

(b1)

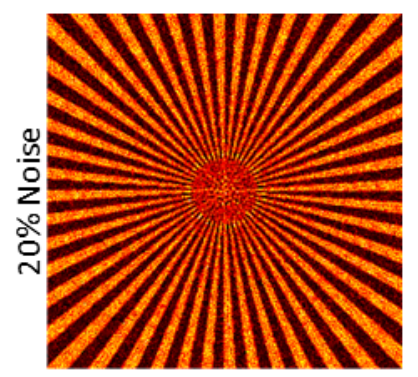

(c1)

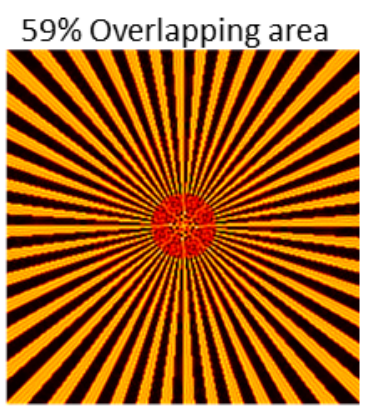

(a2)

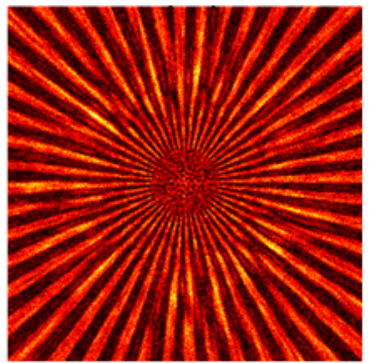

(b2)

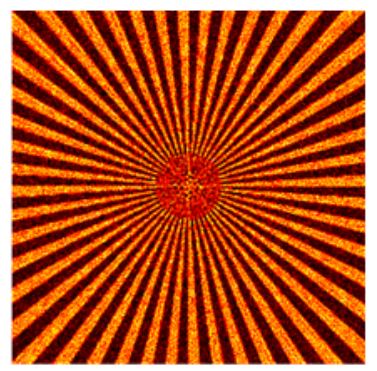

(c2)

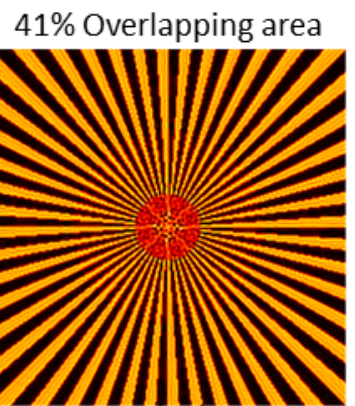

(a3)

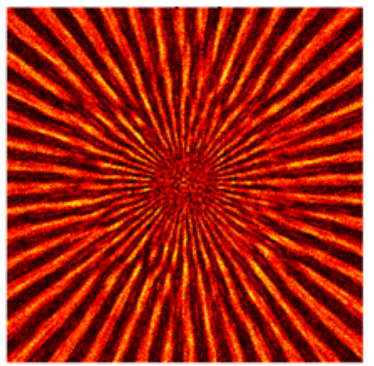

(b3)

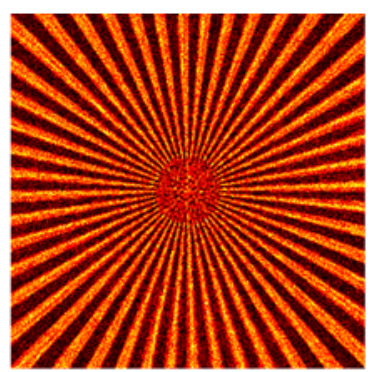

(c3)

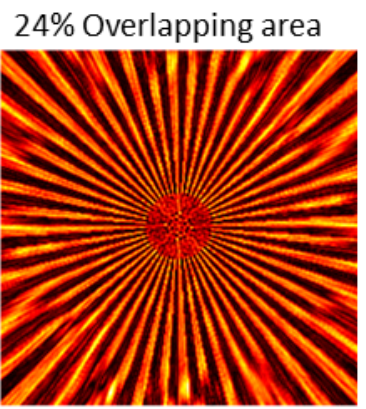

(a4)

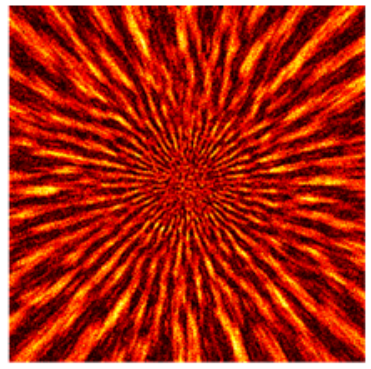

(b4)

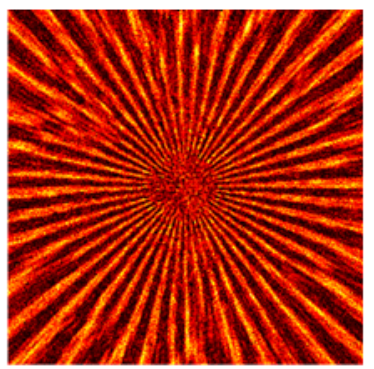

(c4)

Fig. 7. Noise performance of FPM with $20 \%$ additive Gaussian noise level. (a1)-(a4) Images of noise-free, spoke-like sample iterated until convergence with $81 \%, 59 \%, 41 \%$ and $24 \%$ overlapping area in frequency domain, respectively. (b)-(c) Corresponding images of the same sample with $20 \%$ additive Gaussian noise level. (b1)-(b4) Iterating for only once, and (c1)-(c4) iterating until convergence. 
Gaussian noise with a standard deviation of $20 \%$ was introduced into the detection process at CCD. Figures 7(b1)-7(b4) are the recoveries obtained by iterating for only once. While Figs. 7(c1)-7(c4) are acquired by iterating until the solution tends to be stable, where the iterative times are 5, 7, 16 and 29, respectively. From the images obtained via FPM, it is apparent that adding Gaussian noise would not change the basic law discussed above, i.e., the converging speed increases with the growth of the overlapping area. However, the introducing of
Gaussian noise will slow the speed of convergence relative to the situation without noise.

To further study the noises performance of the proposed algorithm, simulations of resolution target were made by adding different amount of noise to the raw image. As is depicted in Fig. 8, different Gaussian noise levels were added to the raw images. When the Gaussian noise is introduced, it is apparent that the resolution decreases as the noise standard deviation increases as shown in Figs. 8(a)8(c). In Fig. 8(d), we plotted the curves of MSE as a

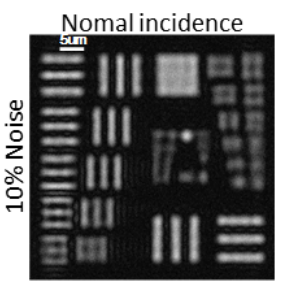

(a1)

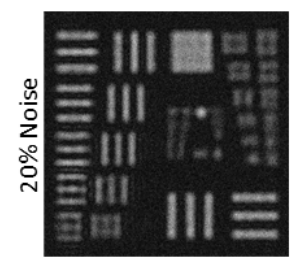

(b1)

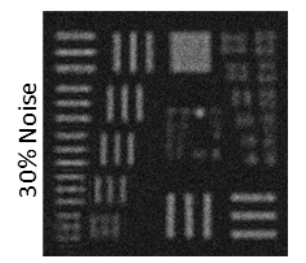

(c1)

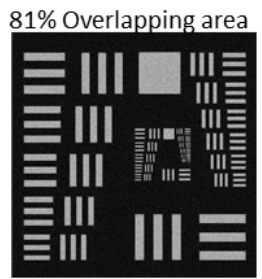

(a2)

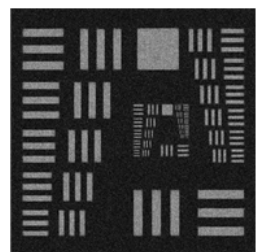

(b2)

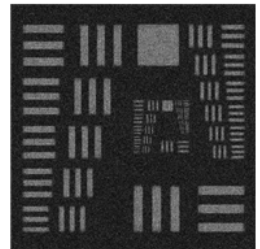

(c2)

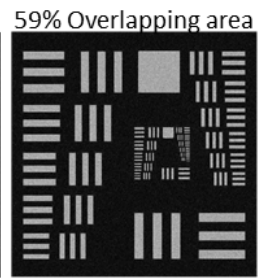

(a3)

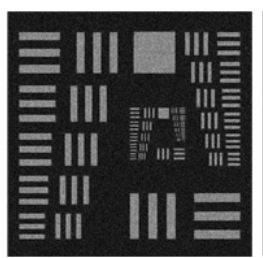

(b3)

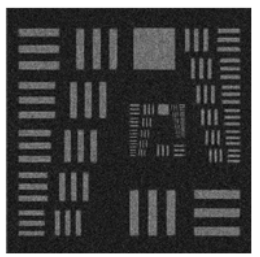

(c3)

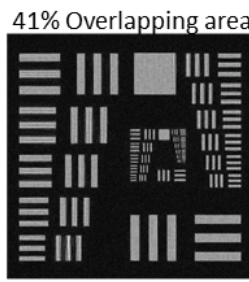

(a4)

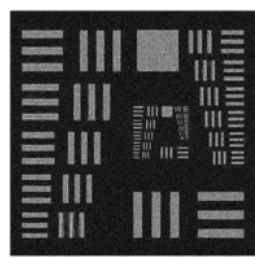

(b4)

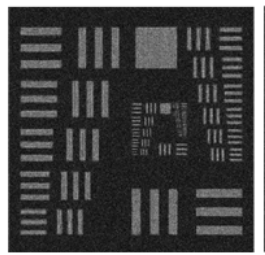

(c4)

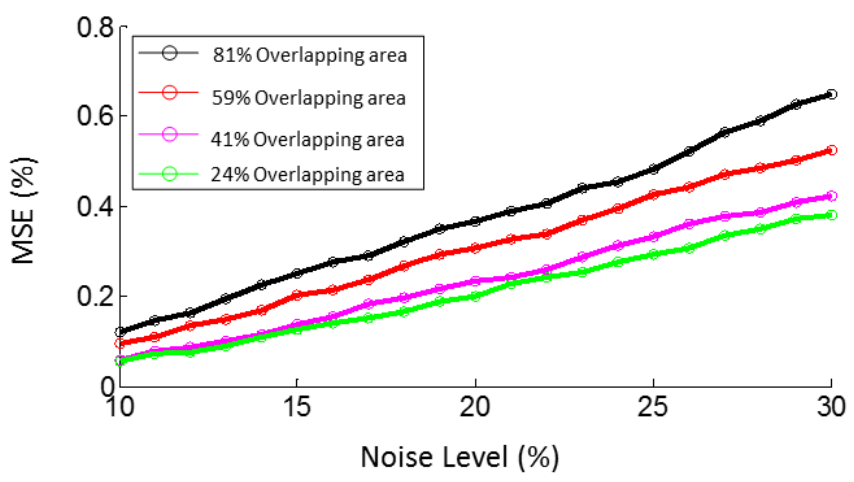

(d)

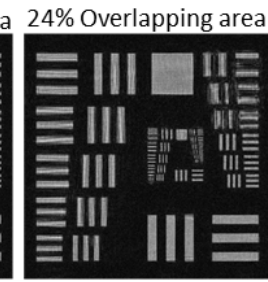

(a5)

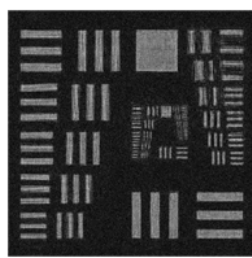

(b5)

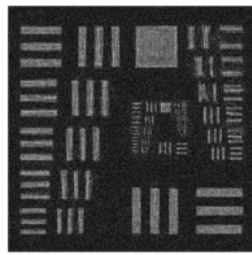

(c5)

Fig. 8. Simulation results under Gaussian noise with different standard deviations. (a)-(c) Images of resolution target corrupted with 10\%, 20\%, and 30\% Gaussian noise levels, respectively. (a1)-(c1) Low-resolution images acquired under normal illumination. (a2)-(a5), (b2)-(b5), and (c2)-(c5) Images with corresponding Gaussian noises reconstructed by FPM until converges with 81\%, $59 \%, 41 \%$, and $24 \%$ overlapping area, respectively. (d) The MSE is described as a function of distinct Gaussian noise levels. Different color lines are corresponding to different overlapping areas in frequency domain. 
Q. Liu et al.

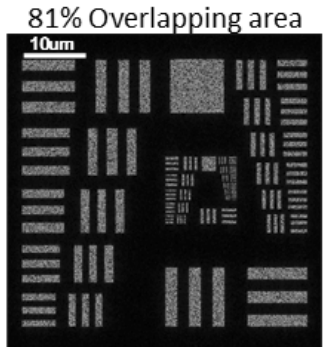

(a1)

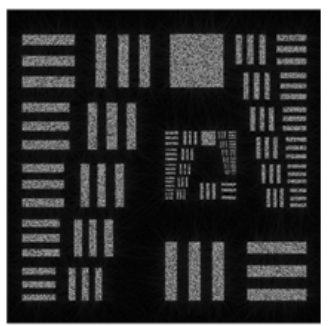

(b1)

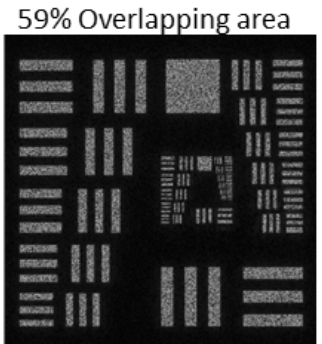

(a2)

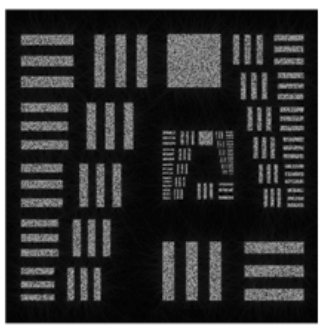

(b2)

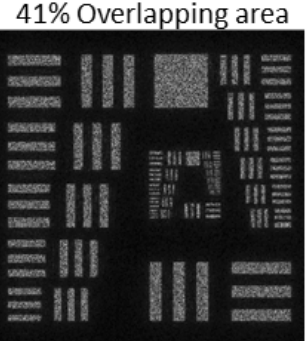

(a3)

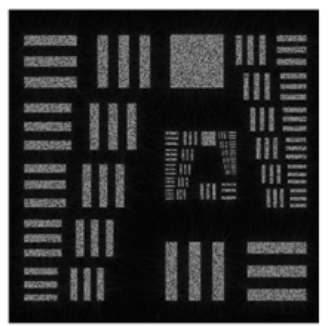

(b3)

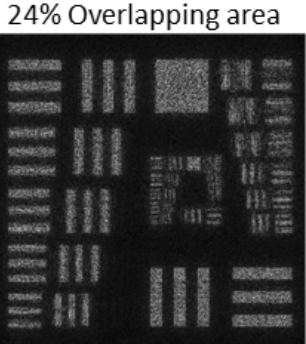

(a4)

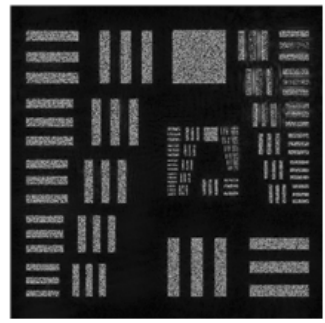

(b4)

Fig. 9. FPM's recovery of resolution target with random phase. (a1)-(a4) Intensity images reconstructed by iterating for once with $81 \%, 59 \%, 41 \%$, and $24 \%$ overlapping area, respectively. (b1)-(b4) Intensity images reconstructed by iterating until converges with the corresponding overlapping area.

function of different noise levels. As we can see that the imaging performance deteriorates linearly with the increasing of noise levels. This means that FPM exhibits good performance with respect to robustness to additive noises. Meanwhile, with the growing of noise levels the distinction of imaging quality brought by different overlapping areas lessens, as is shown in Figs. 8(a3)-8(a4), 8(b3)-8(b4), and $8(\mathrm{c} 3)-8(\mathrm{c} 4)$.

\subsection{Reconstruction of sample with random phase}

In order to investigate the ability of the proposed algorithm to recover the intensity of a given sample, we also simulated the resolution target with random phase. The simulation setting in Fig. 9 is the same as Fig. 5. The iterative times in Figs. 9(b1)-9(b4) are $4,7,11$, and 50, respectively. From the images obtained via FPM, we can see that the relationship between convergence speed and overlapping area sustains if we add a phase to the sample. Meanwhile, the algorithm also exhibits excellent performance of recovering high-resolution intensity image with respect to the sample with phase. Details about the FP's capability of extracting accurate and quantitative phase information from the raw intensity data can be found in Zheng's work. ${ }^{16}$

\section{Conclusion}

We have verified the effectiveness of FPM's algorithm combined with the concept of synthetic aperture. A high-NA objective lens for illumination is used to acquire high spatial information of the sample by providing large illuminating angle, and a low-NA lens for collection is used to capture raw images with wide field of view. The final resolution determined by these two objective lenses is described as Eq. (5). The lateral component $k_{c}$ of oblique incident wave corresponds to a spatial frequency shift via illumination. A high resolution has been acquired in TIR by generating the evanescent field ${ }^{26}$ illuminating the sample with large $k_{c}$ in our group. Besides, applying the characteristic of large lateral wave vector of SPP (surface plasmon polaritons) $)^{27}$ for illumination, there's no doubt that a higher resolution would be achieved. This research will be worthwhile to explore in detail in the future.

A relationship between data redundancy and convergence speed of FPM has been discussed in this paper. The iterative times of the algorithm decreases with the growth of overlapping. The simulation results with $60 \%$ spatial spectrum overlapping acquired by iterating for five times exhibit great imaging quality. The calculating speed is about $5 \mathrm{~s}$ for 100 images, which can be improved by optimizing the program in Matlab and using better 
configuration of computer. We demonstrated that FPM is robust against additive noises via simulating the imaging performance with regard to different Gaussian noises. The presented algorithm of FPM can also recover a high-resolution intensity image from a set of low-resolution images of the sample with random phase.

\section{Acknowledgments}

This work was financially supported by grants from the National Basic Research Program of China (973 Program) (No. 2015CB352003), the National Natural Science Foundation of China (No. 61335003, 61377013,61378051 and 61427818 ), NSFC of Zhejiang province LR16F050001, Innovation Joint Research Center for iCPS (2015XZZX005-01), and Open Foundation of the State Key Laboratory of Modern Optical Instrumentation.

\section{References}

1. E. Rittweger, K. Y. Han, S. E. Irvine, C. Eggeling, S. W. Hell, "STED microscopy reveals crystal colour centres with nanometric resolution," Nat. Photon. 3, 144-147 (2009).

2. E. Betzig, G. H. Patterson, R. Sougrat, O. W. Lindwasser, S. Olenych, J. S. Bonifacino, M. W. Davidson, J. Lippincott-Schwartz, H. F. Hess, "Imaging intracellular fluorescent proteins at nanometer resolution," Science 313, 1642-1645 (2006).

3. M. J. Rust, M. Bates, X. Zhuang, "Sub-diffractionlimit imaging by stochastic optical reconstruction microscopy (STORM)," Nat. Methods 3, 793-796 (2006).

4. E. Mudry, K Belkebir, J. Girard, J. Savatier, E. Le Moal, C. Nicoletti, M. Allain, A. Sentenac, "Structured illumination microscopy using unknown speckle patterns," Nat. Photon. 6, 312-315 (2012).

5. T. M. Turpin, L. H. Gesell, J. Lapides, C. H. Price, in International Society for Optics and Photonics (1995).

6. T. R. Hillman, T. Gutzler, S. A. Alexandrov, D. D. Sampson, "High-resolution, wide-field object reconstruction with synthetic aperture fourier holographic optical microscopy," Opt. Express 17, 78737892 (2009).

7. L. Granero, V. Micó, Z. Zalevsky, J. García, "Synthetic aperture superresolved microscopy in digital lensless Fourier holography by time and angular multiplexing of the object information," Appl. Opt. 49, 845-857 (2010).
8. M. Kim, Y. Choi, C. Fang-Yen, Y. Sung, R. R. Dasari, M. S. Feld, W. Choi, "High-speed synthetic aperture microscopy for live cell imaging," Opt. Lett. 36, 148-150 (2011).

9. J. C. Curlander, R. N. McDonough, Synthetic Aperture Radar; John Wiley \& Sons, New York (1991).

10. Y. Cotte, F. Toy, P. Jourdain, N. Pavillon, D. Boss, P. Magistretti, P. Marquet, C. Depeursinge, "Marker-free phase nanoscopy," Nat. Photon. 7, 113-117 (2013).

11. C. J. Schwarz, Y. Kuznetsova, S. Brueck, "Imaging interferometric microscopy," Opt. Lett. 28, 14241426 (2003).

12. M. Paturzo, P. Ferraro, "Correct self-assembling of spatial frequencies in super-resolution synthetic aperture digital holography," Opt. Lett. 34, 3650-3652 (2009).

13. M. Paturzo, F. Merola, S. Grilli, S. De Nicola, A. Finizio, P. Ferraro, "Super-resolution in digital holography by a two-dimensional dynamic phase grating," Opt. Express 16, 17107-17118 (2008).

14. D. J. Lee, A. M. Weiner, "Optical phase imaging using a synthetic aperture phase retrieval technique," Opt. Express 22, 9380-9394 (2014).

15. G. Zheng, R. Horstmeyer, C. Yang, "Wide-field, high-resolution Fourier ptychographic microscopy," Nat. Photon 7, 739-745 (2013).

16. X. Ou, R. Horstmeyer, C. Yang, G. Zheng, "Quantitative phase imaging via Fourier ptychographic microscopy," Opt. Lett. 38, 4845-4848 (2013).

17. S. Dong, R. Horstmeyer, R. Shiradkar, K. Guo, X. Ou, Z. Bian, H. Xin, G. Zheng, "Aperture-scanning Fourier ptychography for 3D refocusing and super-resolution macroscopic imaging," Opt. Express 22, 13586-13599 (2014).

18. S. Dong, R. Shiradkar, P. Nanda, G. Zheng, "Spectral multiplexing and coherent-state decomposition in fourier ptychographic imaging," Biomed. Opt. Express 5, 1757-1767 (2014).

19. G. Zheng, "Breakthroughs in photonics 2013: Fourier ptychographic imaging," IEEE Photon. J. 6, 1-7 (2014).

20. G. Zheng, X. Ou, R. Horstmeyer, J. Chung, C. Yang, "Fourier ptychographic microscopy: A gigapixel superscope for biomedicine," Opt. Photon. News 25, 26-33 (2014).

21. A. Williams, J. Chung, X. Ou, G. Zheng, S. Rawal, Z. Ao, R. Datar, C. Yang, R. Cote, "Fourier ptychographic microscopy for filtration-based circulating tumor cell enumeration and analysis," J. Biomed. Opt. 19, 066007 (2014).

22. R. W. Gerchberg, "A practical algorithm for the determination of phase from image and diffraction plane pictures," Optik 35, 237 (1972). 
23. A. Den Dekker, A. Van den Bos, "Resolution: A survey," J.-Opt. Soc. Am. A 14, 547-557 (1997).

24. J. W. Goodman, S. C. Gustafson, "Introduction to fourier optics," Opt. Eng. 35, 1513 (1996).

25. P. Gao, G. Pedrini, W. Osten, "Structured illumination for resolution enhancement and autofocusing in digital holographic microscopy," Opt. Lett. 38, 1328-1330 (2013).
26. X. Hao, C. Kuang, Y. Li, X. Liu, "Evanescent-waveinduced frequency shift for optical superresolution imaging," Opt. Lett. 38, 2455-2458 (2013).

27. S. A. Maier, Plasmonics: Fundamentals and Applications; Springer Science \& Business Media, Berlin, (2007). 